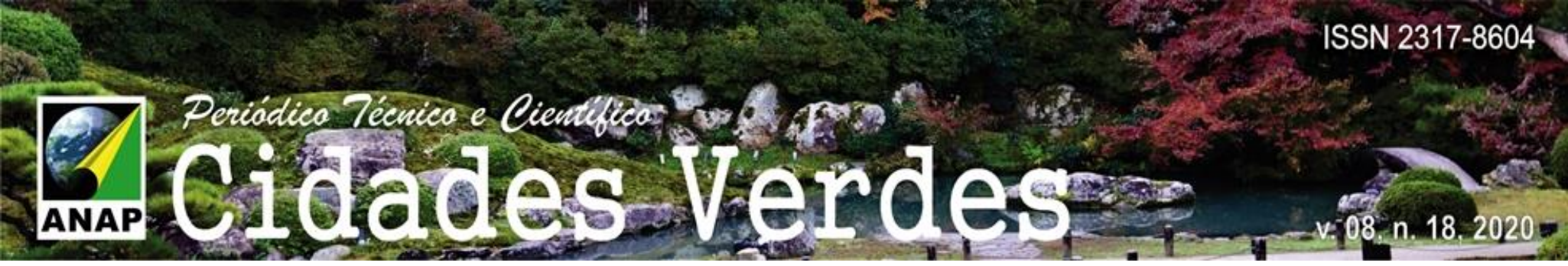

\title{
Retrofit: uma contribuição para a sustentabilidade no caso Citicenter
}

\author{
Retrofit: a contribution to sustainability in the Citicenter
}

Retrofit: una contribución a la sostenibilidad en el caso de Citicenter

Ana Rita Ruescas

Arquiteta e urbanista, Brasil.

arr_arquitetura@outlook.com

Edite Galote Rodrigues Carranza

Professora Doutora, USJT, Brasil. prof.ecarranza@usjt.br

Rodrigo de Paula Ferreira

Professor Mestre, ETEC, Brasil. rodrigo_p_ferreira@hotmail.com 


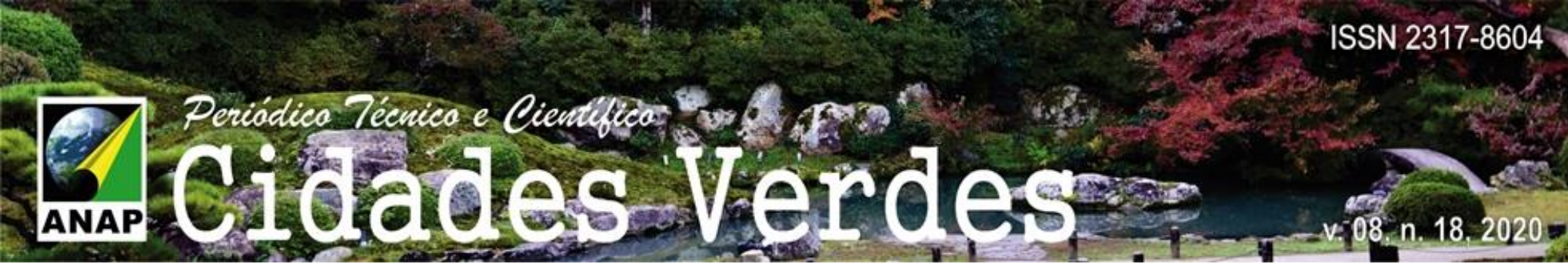

\section{Resumo}

O edifício Citicenter projetado pelo escritório Aflalo \& Gasperini, em 1983, na Av. Paulista em São Paulo, passou recentemente por um processo de atualização através de uma intervenção de retrofit. Em termos atuais, entende-se como retrofit vários procedimentos de intervenção que visam evitar a obsolescência dos edifícios, mediante a aplicação de técnicas e tecnologias atuais; além disso, o retrofit pode contribuir positivamente com as metas estabelecidas na COP 21. No Citicenter, em 2014 à 2016, produtos e equipamentos tecnológicos foram incorporados ao prédio, que em conjunto resultaram na sua atualização e diminuição dos impactos ambientais para a nova fase de vida e uso do edifício. O objetivo do artigo é apresentar como foram aplicadas as estratégias de atualização tecnológica do edifício Citicenter, utilizando o LEED como ferramenta norteadora no processo de integração do retrofit à sustentabilidade. Para o levantamento de dados, foi realizado pesquisa em material bibliográfico - revistas e artigos - e visita técnica ao edifício com acompanhamento do setor de facilities, nas instalações do prédio. Através disto, o artigo demonstra como o retrofit atrelado à sustentabilidade pode ser uma alternativa para o patrimônio edificado da metrópole, evitando que edifícios caiam em desuso, ampliando a longevidade operacional e reduzindo impactos negativos ao meio ambiente, contribuindo com os ODS da Agenda 2030. A partir dos resultados preliminares obtidos, será possível verificar se este tipo de reforma é uma tendência na arquitetura paulistana contemporânea no contexto pós-Acordo de Paris.

Palavras-chave: Retrofit. Sustentabilidade. Arquitetura Paulistana.

\section{Abstract}

The Citicenter building designed by the Aflalo \& Gasperini office, in 1983, at Av. Paulista in São Paulo, has recently undergone an update process through a retrofit intervention. In current terms, retrofit is understood to be several intervention procedures that aim to avoid the obsolescence of buildings, through the application of current techniques and technologies; in addition, the retrofit can positively contribute to the goals established at COP 21. In Citicenter, in 2014 to 2016, technological products and equipment were incorporated into the building, which together resulted in its updating and reduction of environmental impacts for the new phase of building life and use. The objective of the article is to present how the technological updating strategies of the Citicenter building were applied, using LEED as a guiding tool in the process of integrating retrofit to sustainability. For data collection, research was carried out on bibliographic material - magazines and articles - and a technical visit to the building with monitoring of the facilities sector, at the building's facilities. Through this, the article demonstrates how the retrofit linked to sustainability can be an alternative to the built heritage of the metropolis, preventing buildings from falling into disuse, increasing operational longevity and reducing negative impacts on the environment, contributing to the Agenda 2030 SDGs. From the preliminary results obtained, it will be possible to verify whether this type of reform is a trend in contemporary São Paulo architecture in the post-Paris Agreement context.

Keywords: Retrofit. Sustainability. Paulistana architecture.

\section{Resumen}

El edificio Citicenter diseñado por la oficina de Aflalo \& Gasperini, en 1983, en la Av. Paulista en São Paulo, recientemente se sometió a un proceso de actualización a través de una intervención de modificación. En términos actuales, se entiende que la modernización son varios procedimientos de intervención que tienen como objetivo evitar la obsolescencia de los edificios, mediante la aplicación de las técnicas y tecnologías actuales; Además, la actualización puede contribuir positivamente a los objetivos establecidos en la COP 21. En Citicenter, en 2014 a 2016, se incorporaron productos y equipos tecnológicos al edificio, lo que en conjunto resultó en su actualización y reducción de los impactos ambientales para la nueva fase de construyendo vida y uso. El objetivo del artículo es presentar cómo se aplicaron las estrategias de actualización tecnológica del edificio Citicenter, utilizando LEED como herramienta guía en el proceso de integración de la modernización a la sostenibilidad. Para la recolección de datos, se realizó una investigación sobre material bibliográfico (revistas y artículos) y una visita técnica al edificio con monitoreo del sector de las instalaciones, en las instalaciones del edificio. A través de esto, el artículo demuestra cómo la modificación vinculada a la sostenibilidad puede ser una alternativa al patrimonio construido de la metrópoli, evitando que los edificios caigan en desuso, aumentando la longevidad operativa y reduciendo los impactos negativos en el medio ambiente, contribuyendo a los ODS de la Agenda 2030. A partir de los resultados preliminares obtenidos, será posible verificar si este tipo de reforma es una tendencia en la arquitectura contemporánea de São Paulo en el contexto posterior al Acuerdo de París.

Palabras Ilave: Retrofit. Sustentabilidad. Arquitectura paulistana. 


\section{Introdução: Sustentabilidade e o Retrofit do Citicenter}

Localizado na Av. Paulista na cidade de São Paulo, o edifício Citicenter foi projetado em 1983, pelo escritório Aflalo\&Gasperini ${ }^{1}$ e abriga a sede do Citigroup no Brasil, desde 1986. A torre de

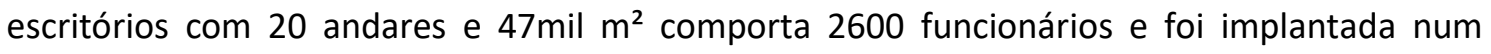
terreno com duas frentes entre a Avenida Paulista (Figura 1) e a Alameda Santos (Figura 2) (DIAS, 2017).

Figura 1. Fachada Av. Paulista

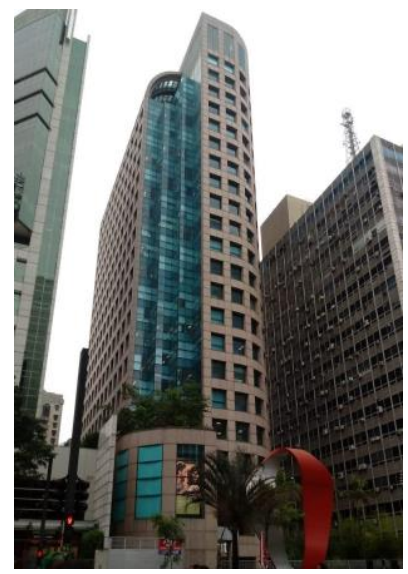

Fonte: FERREIRA, 2020.

Figura 2: Fachada Al. Santos

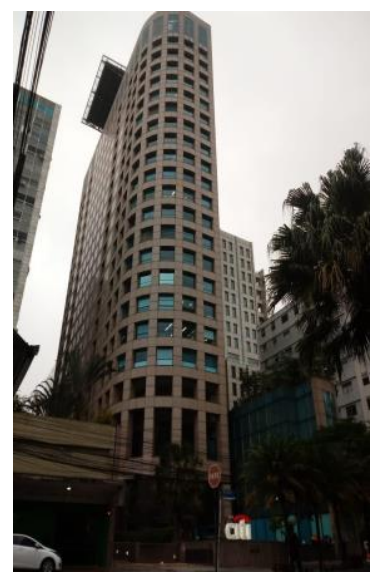

Fonte: FERREIRA, 2020.

A construção do Citicenter na Av. Paulista, coincide com a expansão da cidade de São Paulo para as novas zonas de centralidade corporativa nos anos 1980, seguindo uma nova tendência de concepção arquitetônica, que integra os benefícios da produção industrial e que se evidenciam

\footnotetext{
${ }^{1}$ Aflalo\&Gasperini, escritório de arquitetura fundado em 1962 com a partir da associação dos arquitetos Plínio Croce, Roberto Aflalo e Gian Carlo Gasperini, é especializado em edifícios corporativos, sendo responsável pelos projetos de edifícios como o edifício-sede IBM, o complexo Rochaverá Corporate Towers, o Auditório Cláudio Santoro, entre outros.
} 
em regiões como: Paulista, Pinheiros, Berrini e outras. A nova tendência refere-se ao avanço tecnológico e ampliação da paleta de materiais. Naquela época, seria então uma novidade frente à tendência da arquitetura brutalista paulista, até então hegemônica, e que se caracterizava pela ênfase da plástica do concreto armado aparente (não industrializado), alvenarias de fechamento e esquadrias: de aço e vidro. Em termos tecnológicos, o edifício empregou sistemas de controle de climatização interna dos edifícios, chamados de Heating Ventilation and Air Conditioning (HVAC) com centrais eletrônicas de sistemas de climatização via computadores, precedendo ao conceito Smart Building (Edifício Inteligente).

Segundo Mariano (2013), o conceito de Edifícios Inteligentes surgiu no EUA na década de 1980, com o intuito de utilizar a tecnologia para favorecer os custos de operação dos edifícios com estrutura adequada às necessidades do usuário, a fim de promover conforto, segurança e economia de custos de operação

No Brasil, o primeiro edifício a seguir o conceito de Edifícios Inteligentes foi o Citicenter. Ele foi pioneiro na implantação de tecnologias ao possuir um sistema de monitoramento e controle energético - ainda descentralizado - feito por setores, zonas de iluminância. Também, os elevadores tinham o atendimento por proximidade e o sistema de ar-condicionado era alimentado por produção noturna de gelo, para refrigeração diária, aproveitando as tarifas mais baixas.

Segundo o escritório Aflalo \& Gasperini (2018), o projeto foi concebido a partir da exploração do conceito estrutural em malha, que acarretou em um desenho com planta livre que se encerra nas grelhas estruturais vedada com vidro que se estendem na fachada até os planos curvos das duas frentes do edifício, como é possível se perceber no desenho das plantas (Figura 3-5). No térreo do edifício, o sistema em malha passa por uma transição estrutural que permitiu a criação de áreas com menos influência no térreo, criando um saguão que já abrigou exposições hoje não mais existente, mas que define a criação do espaço semipúblico interligando as duas ruas em desnível.

Figura 3. Implantação do Edifício Citicenter.

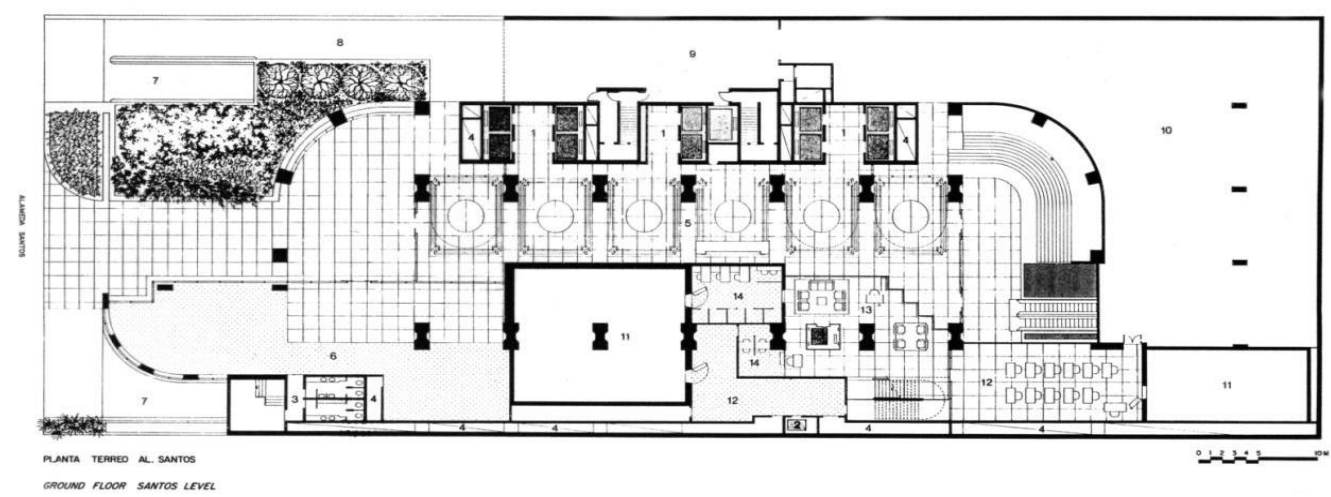

Fonte: AFLALO\&GASPERINI ARQUITETOS, 2020. 
Figura 4. Planta térreo

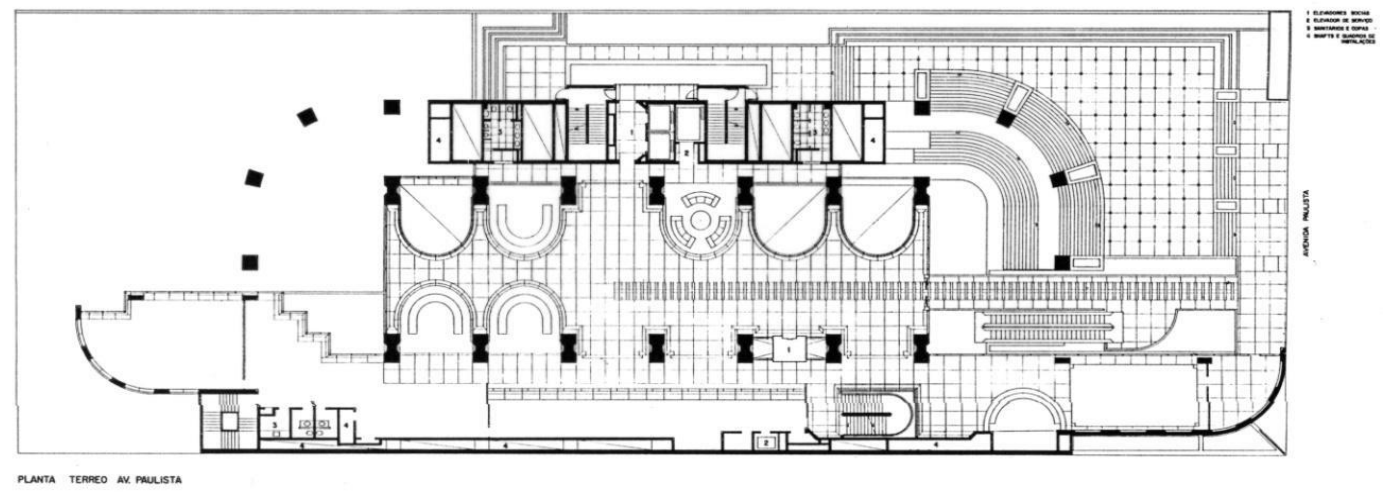

Fonte: AFLALO\&GASPERINI ARQUITETOS, 2020.

Figura 5. Planta sobreloja

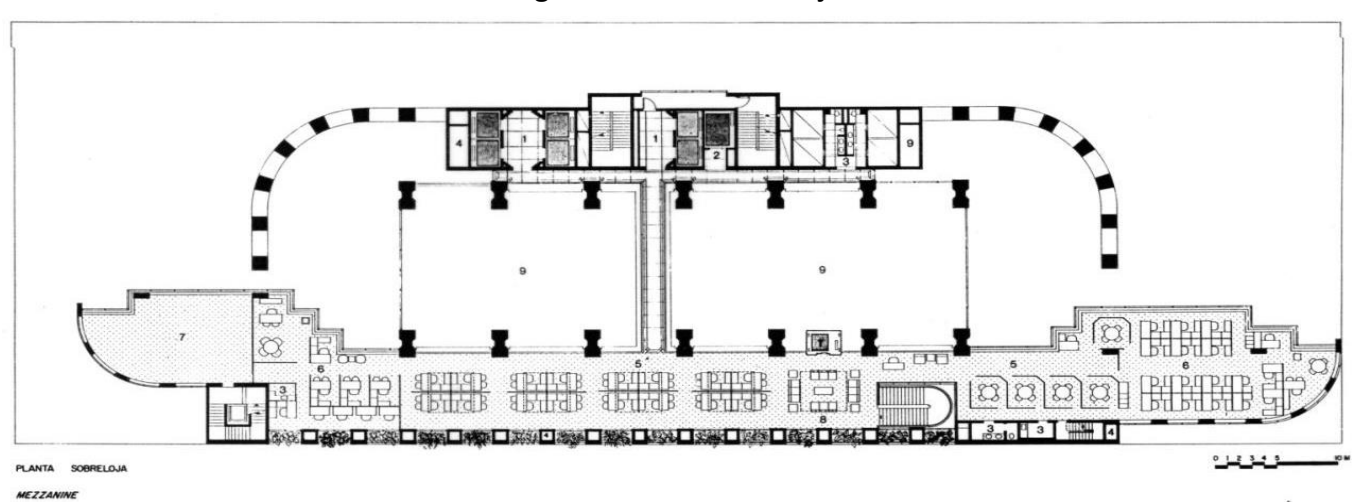

Fonte: AFLALO\&GASPERINI ARQUITETOS, 2020.

As estratégias do projeto do Citicenter tinham como preocupação obter um edifício tecnológico com início de sistemas de automação unidos a um novo modelo de projeto. Apesar da construção ter sido uma revolução na arquitetura, no que tange aos Edifícios Inteligentes, ele foi projetado e construído em um momento que o tema DS (Desenvolvimento Sustentável) não estava em evidência no Brasil. O DS passa a vigorar somente com a conferência no Rio de Janeiro de 1992 (Rio 92/ Eco 92), ou como ficou conhecida Cúpula da Terra, na busca por restabelecer o meio ambiente com a redução nas emissões de gases; mas, somente em 1997, o tema DS passa a ser uma política do Citibank, no mesmo ano em que é feita a elaboração do Protocolo de Kyoto, no Japão, durante a Conferência das Partes 3 (COP 3) (CITIBANK, 2018).

Com a realização da COP 3 , no Japão, os organismos internacionais tomaram uma nova posição com relação às questões ambientais, embora houvesse um conflito entre União Europeia e Estados Unidos. Nessa conferência foi criado o Protocolo de Kyoto. Um documento legalizado que sugere a redução de gases do efeito estufa (cujas metas 
são de 5,2\%) e para que fosse aprovado, os países desenvolvidos deveriam aceitar o acordo, pois eles correspondiam a maior parte das emissões de gases poluentes da atmosfera (MINISTÉRIO DO MEIO AMBIENTE, 2018).

No mesmo ano 1997, a Citi Foundation 60 amplia as atividades socioambientais com projetos de forma planejada, monitorada e sistemática que leva o Citibank em 2001 ao ingresso no índice Dow Jones de Sustentabilidade 61(DJSI), passando a fazer parte também do FTSE4Good Index Series 62 (índice da FTSE). Em 2003, ao participar da criação e adoção dos Princípios do Equador, um marco para o banco em direção ao conceito de DS, o banco passa a responder sobre emissões de gases de efeito estufa.

É provável que o Protocolo de Kyoto tenha influenciado às ações do Banco e direcionado funcionários a criarem o programa Citi Esperança, ainda em 1997, no Rio de Janeiro. O objetivo do programa era cooperar com ações socioambientais nas áreas de atuação do Citibank nas comunidades locais. (CITIBANK, 2018).

Assim, com a implantação da política de análise de risco socioambiental, o banco direcionou um setor específico em 2006 para debater questões sobre a Responsabilidade Social Empresarial (RSE) discutindo métodos que unifiquem a sustentabilidade a estratégias de negócios, dando origem ao Relatório de Sustentabilidade (RS) publicado anualmente desde 2008 (CITIBRASIL, 2008).

Com a publicação do RS, o Citibank passa a mapear as ações realizadas e desenvolver estratégias que vão ao encontro dos DS e promove ações de capacitação sobre práticas sustentáveis entre funcionários e colaboradores, principalmente sobre o efeito estufa, considerado um dos principais impactos negativos causados pelo consumo no banco.

Neste contexto, após 30 anos de operação na Avenida Paulista, surge a iniciativa do Citibank de rever as instalações do edifício Citicenter em busca de resultados. Em consonância às políticas do banco, o edifício sede na Avenida Paulista passa por um retrofit entre 2014-2016, a fim de torná-lo um edifício sustentável (DIAS, 2017).

\section{Retrofit: A intervenção no edifício}

O retrofit do Citicenter ocorreu no mesmo período em que no cenário internacional ocorre a COP 21, a qual as nações firmaram o Acordo de Paris, inclusive o Brasil como signatário que ratificou o acordo em setembro de 2016 (BRASIL, M. M. A., 2018). Neste contexto, a intervenção no edifício veio em direção ao compromisso firmado na COP 21 e pode ser entendido como um retrofit consoante ao conceito de ACV por compreender a sustentabilidade junto as técnicas de atualização do edifício.

A saber: O retrofit é uma palavra criada a partir da junção da palavra latina retro e da palavra inglesa fit, cujo significado pode ser entendido como remodelação ou atualização do edifício (CBCS, 2013). Segundo a Associação Brasileira de Normas Técnicas ABNT NBR 15575 o retrofit pode ser entendido como: 
Retrofit é a remodelação ou atualização do edifício ou de sistemas, através da incorporação de novas tecnologias e conceitos, normalmente visando a valorização do imóvel, mudança de uso, aumento da vida útil e eficiência operacional e energética (ABNT, NBR $15.575,2013$, p.9-10).

Segundo o engenheiro Eduardo Qualharini (2004), o retrofit é um estudo complexo dos aspectos que constituem a técnica, com procedimentos específicos e individuais para cada intervenção, porque os rumos propostos à obra são norteados pelas condições inerentes da edificação, que demanda de um Pré-Diagnóstico para se avaliar a viabilidade de aplicação da técnica retrofit e um Diagnóstico para investigar o grau de intervenção possível.

Contudo, Ferreira (2020) discute que, mediante a especificidade de Projetos e Processos inclusos na aplicação do retrofit, a técnica deve ser aplicada junto à metodologia de Avaliação do Ciclo de Vida (ACV). Assim, permite análise de impactos ambientais de diversos produtos, podendo ampliar consideravelmente o desempenho da edificação perante ao combate dos impactos ambientais: desde os impactos já ocasionados até os que vão ocorrer durante a intervenção e na operação dos edifícios, contribuindo com as metas para o Desenvolvimento Sustentável na Agenda 2030.

Portanto, podemos entender que o retrofit do edifício Citicenter Center é um retrofit consoante à $\mathrm{ACV}$, na medida em que as técnicas e tecnologias aplicadas tem a intenção de realizar melhorias na construção existente, além de contribuir com a sustentabilidade.

Algumas Organizações têm contribuído para o norteamento da aplicação do retrofit com intenções sustentáveis, como o caso do U.S. Grenn Building, no Brasil: Green Building Council, uma organização não governamental que trabalha a favor de uma plataforma para tornar as construções sustentáveis, e chancela um certificado em níveis, de acordo com uma somatória de pontos que se referem às ações abarcadas.

O Citicenter foi o primeiro edifício da Avenida Paulista, em São Paulo, e o primeiro Citi na América Latina, a receber a certificação LEED na categoria de prédios já construídos. Oferecida pelo Green Building Council Brasil (GBC Brasil), a certificação valida um conjunto de práticas voltadas à operação e à manutenção sustentável.

Na sequência abordaremos as estratégias que foram usadas no retrofit no Citicenter, analisando os resultados obtidos nos quesitos da certificação LEED.

\section{Discussão: estratégias de retrofit}

O Retrofit do edifício Citicenter foi realizado em consultoria com o Centro de Tecnologia de Edificações (CTE), e executado por empresas específicas às áreas, de elétrica, hidráulica, climatização, luminotécnica, paisagismo e telhado verde (DIAS, 2017).

$\mathrm{Na}$ modalidade LEED Operação e Manutenção $(\mathrm{O}+\mathrm{M})$ se exige que o edifício passe por reavaliação a cada cinco anos (LEED V4, 2014). O que é possível compreender que o retrofit não 
tinha intenção de se limitar as ações durante a intervenção, mas principalmente a de criar uma base de controle de dados que possibilitam o gerenciamento do desempenho e possibilita-se ações preditivas de manutenção do prédio.

Dois fatores contribuíram fundamentalmente nas ações do retrofit: primeiro, a sustentabilidade que já estava em curso como política do banco, assim algumas ações de intervenção haviam iniciado, sendo necessário somente a implantação do sistema de gerenciamento dos dados Building Management System (BMS) (Sistema de Gestão do Edifício) para as ações de operação; segundo, o Citibank já se utilizava de processos de licitação para garantir a contratação adequada de fornecedores.

Segundo o relatório CitiBrasil (2016), as empresas participantes do processo das áreas de elétrica, hidráulica, climatização, luminotécnica, paisagismo e telhado verde, responderam a um Questionário de Responsabilidade Corporativa (QRC), que aborda o tema sustentabilidade unido a participação em um plano de capacitação orientado pela Declaração de Princípios a Fornecedores (DPF), documentos que garantem a seguridade diante de três temas: práticas empresariais éticas; direitos humanos no local de trabalho; sustentabilidade ambiental, bem como o cumprimento da Política de Seleção e Gerenciamento de Fornecedores com o objetivo de estabelecer diretrizes socioambientais, atendendo as Resoluções no 4.327/14, do Banco Central do Brasil, de 25 de abril de 2014.

A intervenção realizada ao edifício acumulou 51 pontos no checklist da categoria $0+M$ em 6 áreas de avaliação das 9 possíveis recebendo o nível Silver (Prata) de certificação. Sendo assim, a análise dos resultados segue a ordem das áreas de avaliação estabelecidas pela certificação LEED.

\section{Terreno Sustentável (SS):}

No terraço da agência anexa ao edifício foi implantado um terraço verde (Figura 8-9) com 526 árvores e aproximadamente 80 espécies nativas da Mata Atlântica. No acesso pela Alameda Santos, criou-se uma parede verde, totalizando $500 \mathrm{~m}^{2}$ de vegetação que reconstituem à Mata Atlântica que existia na área anos atrás (CITIBRASIL, 2016). A estratégia utilizada foi resgatar a diversidade ecológica em meio a construção (Dias, 2017).

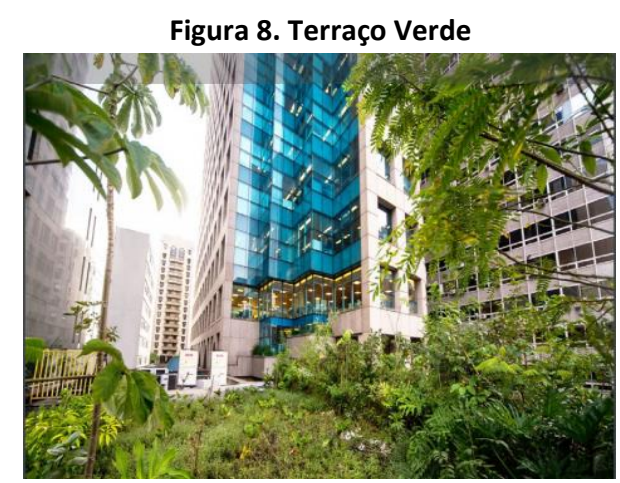

Fonte: DIAS, 2017. 
Figura 9. Terraço Verde visto da Avenida Paulista

\section{Uso racional da água (WE):}

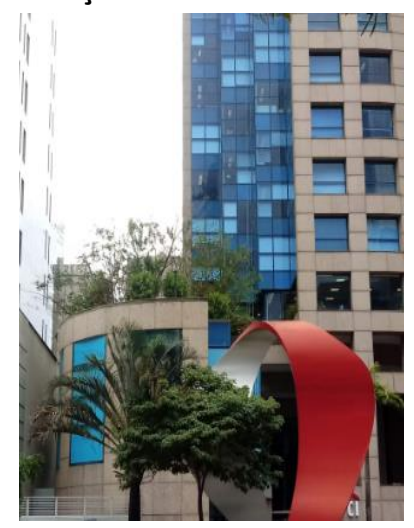

Fonte: FERREIRA, 2020.

A redução do consumo de água do edifício concerne a três estratégias: primeira a implantação do sistema de bombeamento das águas armazenadas no subterrâneo do edifício, que são utilizadas na linha de serviço durante a operação do edifício, junto ao sistema de uso de águas pluviais; segunda, a instalação de redutores de pressão nas torneiras dos banheiros; terceira; a perfuração de um poço em 2016 com capacidade de extração de água em $2.200 \mathrm{~m}^{3} / \mathrm{mês}$ e instalações para atendimento em $100 \%$ do edifício, com vistorias semanais de empresa específica para controle de qualidade da água para consumo. Segundo dados do CitiBrasil (2016, p.30), o consumo de água em 2015 foi de $47.917 \mathrm{~m}^{3}$, já em 2016 totalizou $50.762 \mathrm{~m}^{3}$, representando $5,9 \%$ de acréscimo. No mesmo ano, o Citicenter extraiu $22.227 \mathrm{~m}^{3}$, reduzindo o consumo de água potável no equivalente a $43,8 \%$ do consumo do ano.

Neste item, o retrofit atingiu uma redução de $30,84 \%$ no uso de água potável em ambientes fechados e em $63 \%$ o uso de água potável em áreas verdes atendendo também a critérios específicos implantando sistemas de medição e controle de águas (GBIG, 2018).

\section{Energia e atmosfera (EA):}

Dentro desses parâmetros, o sistema de iluminação foi substituído por lâmpadas LED com sensores que ajustam a intensidade de iluminação artificial à incidência natural. Como estratégia de redução de consumo em operação, o edifício passa por programação de desligamento parcial do sistema de iluminação a partir das 19h, sendo ativos somente em casos específicos previamente informado e se autorizado pela área de facilities (CITIBRASIL, 2014). Além disso, o sistema de condicionamento de ar é utilizado com sensores que se ajustam a temperatura dos pavimentos, evitando a superutilização do sistema. Todas as funções de energia do edifício, exceto a do o sistema de elevadores conectados ao BMS (transmissor de dados simultâneo de consumo e operação de cada equipamento), possibilitou ao Citicenter o gerenciamento e 
operação em área centralizada do consumo energético do edifício, não sendo mais dividido em zonas de controle.

Outra ação como pré-requisito da categoria da certificação LEED foi realizar uma auditoria energética Lv.2, conduzida pela Sociedade Americana de Engenheiros de Aquecimento, Refrigeração e Ar Condicionado (ASHRAE), cujos resultados são utilizados como base para continuidade das estratégias de redução energética (Dias, 2017). Ainda foram realizados processos de conscientização à população do Citicenter sobre a importância do uso consciente da energia.

Segundo o CitiBrasil (2016, p.30), do ano de 2015 para 2016 o consumo de energia no edifício resultou em 1,3\%, passando de $77.289 \mathrm{GJ}$ para $76.284 \mathrm{GJ}$ e 7,3\% se comparado à 2014. Economia considerável, se levarmos em conta o fato de o edifício já ter sido projetado com sistema de gerenciamento de consumos.

Para redução das emissões de $\mathrm{CO} 2$, a diminuição foi em função da substituição da frota de veículos por automóveis a Gás Natural Veicular (GNV), com 16,9\% em gasolina, 18,5\% em diesel e 19,6\% em etanol. Contudo, houve o aumento do GNV em 11,5\%, que ainda está em avaliação para substituir os de maiores emissões (CITIBRASIL, 2016, p.30).

Com a ferramenta Energy Star², o Citicenter conquistou a classificação 80 pontos, ou seja, a redução de $30 \%$ de energia.

\section{Materiais e recursos (MR):}

Para a redução do volume de lixo evitando descartes incorretos, foi implantado no edifício pontos de coleta para seleção dos materiais de descartes. Contudo, o edifício não promove ações internas de reciclagem, somente as direcionam a empresas específicas que fazem a reciclagem de produtos. Para tal, o gerenciamento implantou um setor de monitoramento de resíduos que monitoram a qualidade de sua a separação. Além da coleta seletiva, outra estratégia foi a instalação de purificadores de água nos escritórios situados na área central dos pavimentos com o intuito de eliminar o uso de garrafas plásticas.

Segundo dados do Citibank, o edifício calcula que $47 \%$ dos resíduos gerados estão aptos a serem encaminhados à reciclagem (DIAS, 2017, p 15). Em 2015, foram coletadas 285,68t de resíduos e em 2016, 221,4t, que correspondem a uma redução de $22,5 \%$ no volume. Do total, foram selecionados 144,7t de resíduos orgânicos, 59,9t de papel, 41,2t de resíduos eletrônicos, 15,6t de plásticos e polímeros e 1,2t de resíduos (lâmpadas encaminhadas para incineração) (CITIBRASIL, 2016, p.30).

\section{Qualidade do ambiente interno (EQ):}

Nesta área, o projeto original comprovou que o Citicenter possui iluminação natural em $50 \%$ dos espaços ocupados e fachadas de qualidade (GBIG, 2018), ou seja, a solução estrutural

\footnotetext{
2 ENERGY STAR Portfolio Manager ${ }^{\circledR}$, um software usado para medir e identificar o consumo de energia e emissões de gases de efeito estufa. A certificação é fornecida para edifícios que atingem no mínimo 75 pontos de um total de 100.
} 
proposta pelos arquitetos em grelha permitiu uma independência dos sistemas estruturais com os elementos de vedação, permitindo o envidraçamento em todas as elevações do edifício e mantendo uma relação visual dos usuários do edifício com a paisagem externa. Desse modo, a qualidade arquitetônica do projeto original do Citicenter contribui favoravelmente ao conforto e bem-estar de seus usuários e ocupantes do edifício, pois atende aos requisitos desta categoria (LEED O+M V4, 2014), o que resultou em 2 pontos na somatória.

\section{Resultados}

Sendo assim, todas as ações apresentadas acima demonstram como a técnica do retrofit concomitante as estratégias de ACV, proposta pela certificação LEED para a longevidade da operação do edifício, integra a tendência de estratégias sustentáveis para edifícios existentes. Os números de economia de água, $43,8 \%$, de energia, $7,3 \%$ e de resíduos $22,5 \%$, entre outros, revelam que as intervenções realizadas foram positivas.

\section{Considerações finais}

Este artigo demonstrou algumas estratégias de intervenção possíveis de retrofit na arquitetura, explorando a aplicação da intervenção no caso do edifício Citicenter e os resultados favoráveis após o processo no prédio. Desta forma, conclui-se que o Citicenter, como sede de um banco internacional, segue os parâmetros e orientações internacionais do Citibank. Assim, o projeto original procurou demonstrar uma relação social, com permeabilidade visual dos usuários do edifício e espaços de fruição pública, atendendo a políticas internas do banco à época. Contudo, o edifício não foi originalmente projetado para ser uma arquitetura sustentável - como entendemos hoje. Porém, no contexto pós COP 21, de 2015, quando se firmou os 17 Objetivos de Desenvolvimento Sustentável (ODS) da Organização das Nações Unidas, ao menos 4 podendo ser aplicados à arquitetura e a evolução nas políticas de operação do Citibank, o retrofit veio ao encontro da necessidade da atualização e integração da sustentabilidade ao edifício. Neste caso, a certificação LEED O+M como plataforma mundial, foi uma ferramenta norteadora adequada para as intervenções da reforma, colocando o edifício abordado como uma referência sustentável em meio às edificações novas ou reformadas da arquitetura paulistana e pode ser entendido como um "Case" de sucesso. Mediante os dados obtidos neste estudo, é possível afirmar que o retrofit orientado pela certificação LEED O+M, contribuiu com a redução da "Pegada Ecológica", atende às metas estabelecidas no Acordo de Paris, preserva o patrimônio construído, contribui com a meta estabelecida na Agenda de 2030 e, talvez, seja uma tendência para a arquitetura contemporânea paulistana. 
